\title{
A comparison between the X-ray variable Sun and solar-like main sequence stars
}

\author{
G. Micela ${ }^{1}$ and A. Marino ${ }^{2}$ \\ 1 INAF, Osservatorio Astronomico di Palermo, Piazza del Parlamento 1, 90134 Palermo, Italy \\ 2 Dipartimento di Scienze Fisiche e Astronomiche - Università di Palermo, Piazza del Parlamento 1, 90134 Palermo, Italy
}

Received 7 January 2003 / Accepted 21 March 2003

\begin{abstract}
We analyze the time variations of the solar X-ray luminosity observed with Yohkoh/SXT with the aim to compare the $\mathrm{X}$-ray variability of the Sun with that of the other solar-like main sequence stars as function of the relevant time scales. Since the observational set-up and strategies used to observe the Sun differ from those used for the other stars, we have explored the solar X-ray variability properties starting from the available solar data, trying to reproduce the observational procedures adopted for the stars. We have quantified how the solar variability amplitude increases with the explored time scales and found that solar-cycle variability can contribute at most up to $60 \%$ to the spread observed in nearby solar-like star X-ray luminosity distribution functions. The comparison between the Sun and the nearby stars is consistent with a scenario in which a fraction of moderately active stars $\left(\bar{L}_{x}<10^{28} \mathrm{erg} / \mathrm{s}\right)$ have X-ray variability similar to the Sun, while more active stars lack solar-like cyclic coronal activity.
\end{abstract}

Key words. Sun: corona - stars: coronae - X-rays: stars

\section{Introduction}

The solar corona is often used as a paradigm to understand stellar coronae. The possibility to observe the Sun in detail in space and in time allows us to understand how the solar corona is built and to extrapolate these results to the other stars.

The Sun is highly variable when observed in X-rays, with large amplitude variations observed over a range of time scales, depending on the nature of mechanism producing the variability. Roughly, variations on short time scales (less than or of the order of a few hours) are associated with flares, medium scale variations (months) are associated with the evolution of active regions and with rotational modulation, and long term (years) variations are associated with the solar cycle.

If the Sun is a typical main sequence late-type star, we expect to observe the same variations on other main sequence stars, but in X-ray bandpass normal stars appear less variable than the Sun. With the notable exceptions of some big flares observed on $\mathrm{dMe}$ or on very active stars (see e.g. Pallavicini et al. 1990; Schmitt 1994), only variations within a factor 2-3 are observed in normal main sequence stars, while the Sun shows variations up to more than one order of magnitude during the cycle (see Stern 1998 for an extensive discussion). Hempelmann et al. (1996), using ROSAT All Sky Survey observations of stars with known Ca II cycles from the Mount Wilson $\mathrm{Ca}$ II $\mathrm{H}$ and $\mathrm{K}$ program suggested that these stars can

Send offprint requests to: $\mathrm{G}$. Micela, e-mail: giusi@astropa.unipa.it have also X-ray cycles. Unfortunately their result is marginal and based on a sample selected for having Ca II cycles and not on an unbiased sample.

Moreover the comparison between the solar and stellar coronae is not straightforward, as it involves a number of problems. First of all, the assumptions that the Sun is representative of all the other stars is not a priori justified. In particular the most active stars have X-ray luminosity up 10-100 times larger than the Sun, and for them the solar paradigm could be inadequate (e.g. Drake et al. 2000). Another difficulty is that we observe the Sun with a methodology completely different from that adopted for stars. Paradoxically we have never observed the Sun as a star in the soft X-ray band. For example the Sun and the stars are observed in different energy bands and solar variability properties are strongly dependent on energy and time scales (Kreplin 1970). An effort to study the solar corona as if it were a stellar one has been done by Peres and collaborators (Peres et al. 2000 and papers of this series) that have developed a technique that makes use of Yohkoh data to simulate stellar-like observations. They take advantage of the spatial resolution of the Yohkoh/SXT instrument and of its two filters to build a map of the effective temperature and emission measure distribution of each image pixel of the Sun. Using this map the authors synthesize the emitted spectra and fold them through the response of X-ray detectors used for extra-solar observations, analyzing the result as a typical stellar observation. In this way they can study, for example, as the Sun would appear when observed as a star during different stages of the cycle, 


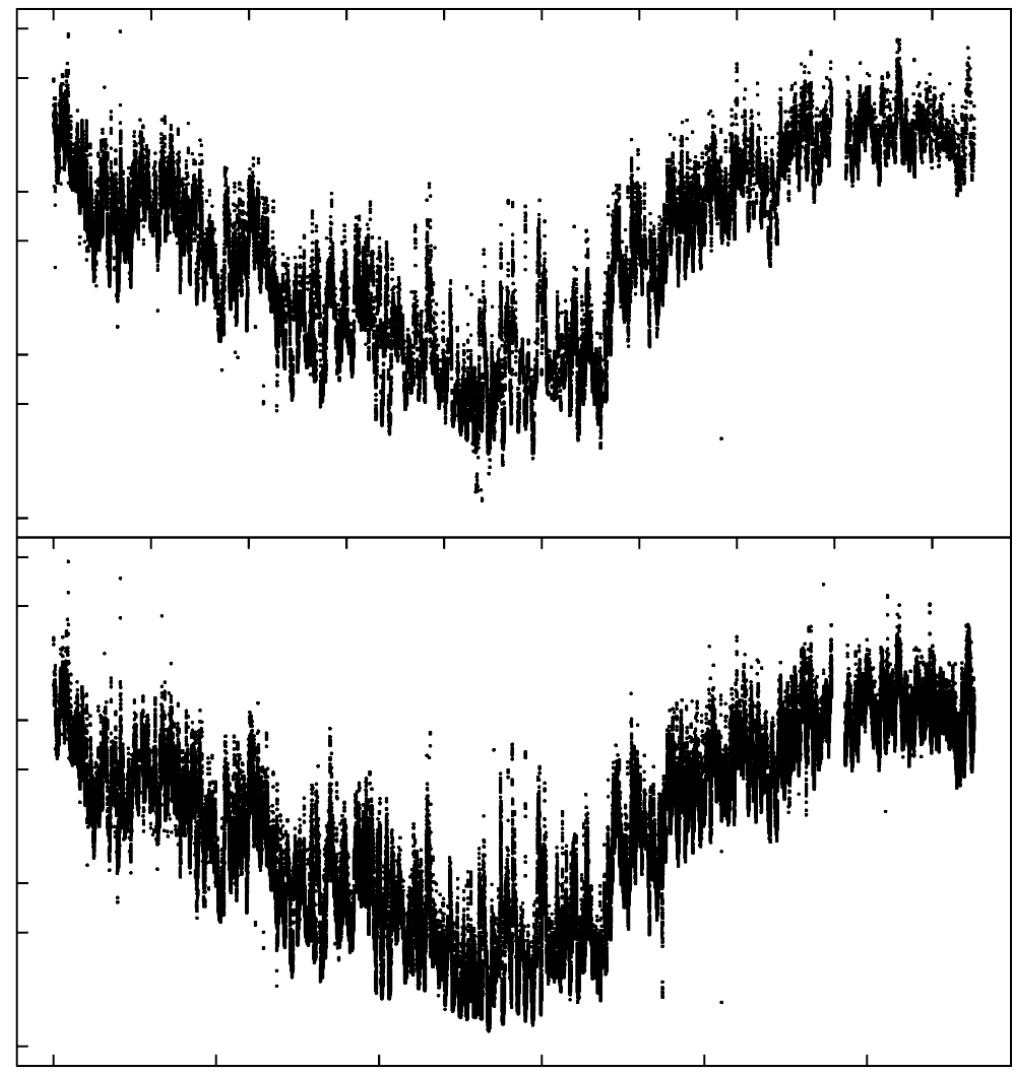

Fig. 1. Light curve of the Sun observed with Yohkoh with the Al (top panel) and AlMg filter (bottom panel). Time is measured starting from January 1st 1992. Note the minimum and the maximum observed on 1996 and 2000, respectively.

what is the effect of rotational modulation, what are the properties of individual coronal components such as active regions, flares, etc.

In this paper we use a less sophisticated approach, which makes use of all the data publically available in the SolarSoftWare (SSW) database ${ }^{1}$ in order to understand what are the properties of solar X-ray variability when the Sun is observed as a star, and how these properties compare with stellar observations.

In this comparison we will try to take into account, wherever possible, the differences in observational procedures, in the explored time scales, in the energy bands, and in the temporal sampling.

In the next section we will discuss the solar data we use and their statistical properties from the stellar point of view. In Sect. 3 we will discuss the effects of temporal sampling on the observed variability. In Sect. 4 we will compare our results with the observations of nearby stars. Our main results are summarized in Sect. 5.

\footnotetext{
${ }^{1}$ available on http://sohowww. nascom.nasa.gov/solarsoft and maintained by Robert Bentley, MSSL, and Samuel Freeland, LMSAL.
}

\section{Data}

We have analyzed the Yohkoh/SXT synoptic data obtained both with the Al $1265 \AA$ filter (in the following Al) and with the filter made by a sandwich of $\mathrm{Al}, \mathrm{Mg}, \mathrm{Mn}$, and $\mathrm{C}$ (AlMg hereafter). The bandpasses of the two filters are very similar, the latter slightly harder than the former, and both have most of the effective area in the range between 5 and $17 \AA(0.73-2.5 \mathrm{keV})$. The synoptic observations of Yohkoh/SXT consist of a large number of observations, with time separations ranging from few minutes to hours, with most of the observations separated by 10-15 min. Only in few cases there are whole days without observations. These "holes" can be due to instrumental problems or to the occurrence of some flares. In this latter case the instrument switches on a different observational setup specially suited to observe flares. This observational strategy introduces a bias against the largest flares that will be discussed in the following.

The data we use are reported in Fig. 1 for the two filters. The reported quantity is Digital Number per pixel per second and is proportional to the X-ray photon flux; it is computed from the histograms of full frame desaturated images (Morrison 1994). In the following we refer to this quantity as "X-ray flux". The zero time that we have considered is 


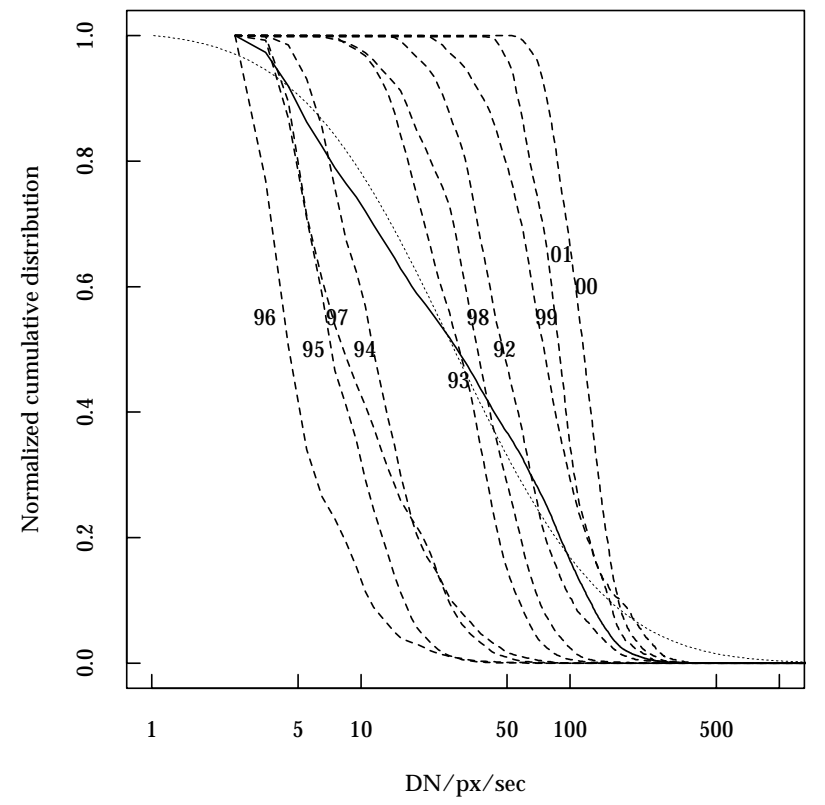

Fig. 2. Intensity cumulative distribution observed on the Sun with the AlMg filter during the cycle (solid line). Dashed lines are the distributions observed in individual years. Thin dotted line is the log-normal distribution with the same standard deviation than that of the solar distribution observed during the cycle.

January 1st 1992 and the series spans about 10 years. Data cover about one solar cycle allowing the study of variability up to this time scale. In total there are 5203 data points obtained with the $\mathrm{Al}$ filter and 60228 with the $\mathrm{AlMg}$ one. Results derived in the following relative to the AlMg filters are fully consistent with those derived from $\mathrm{Al}$ observations.

To understand more quantitatively the statistical properties of the X-ray Sun as a star we have built the distributions in time of X-ray flux of the Sun as observed by Yohkoh. The distribution relative to the AlMg filter is shown in Fig. 2 (the distributions obtained with the Al filter have the same characteristics). If we observe a large sample of stars with the same properties as the Sun, we expect to observe a stellar X-ray luminosity distribution with the same shape of the time distribution of the Sun, since each stellar observation should be equivalent to one solar observation in a different moment of the cycle. The spread observed in the solar distribution is the expected contribution to the spread observed in the stellar X-ray luminosity distributions due to the variability (of the same nature of that observed in the Sun).

The solar curve relative to the full data set, covering the complete solar cycle, (solid line in Fig. 2) can be described by a log-normal distribution with $\sigma=0.52$ dex for the Al filter and 0.58 dex for the AlMg one. Comparing these numbers with the standard deviations obtained by Schmitt (1997) for Fand G-type stars within $13 \mathrm{pc}(0.96$ and $0.82 \mathrm{dex}$ for the stars in the $0.3<B-V<0.6$ and $0.61<B-V<0.82$ range, respectively), we find that about $60 \%$ of the spread observed in the X-ray luminosity in nearby solar-like stars can be explained by solar-like variability. Indeed the derived fraction is an upper limit to the contribution to the spread, since the X-ray luminosity distributions computed by Schmitt for the stars refer to the ROSAT/PSPC band, softer than the Yohkoh/SXT bandpass. Since in the Sun the hard band variations are more pronounced that the soft ones (Kreplin 1970), we can safely conclude that at most $60 \%$ of the observed spread in stellar X-ray luminosity can be reproduced by solar-like variability. Note that the standard deviation, derived from the solar cycle, is independent of the cycle length, while it depends on the amplitude.

The standard deviation observed in each year, in which variations are dominated by short and medium term variability (with small effects due to the cycle), is on average about half of that observed in the entire cycle. We conclude that an $\mathrm{X}$-ray variability by a factor of two is due to short and medium term variability, while the presence of the solar cycle increases this variability to a factor of three to four.

\section{Time scale effects}

In this section we study how the observed variability changes as a function of the time scale we explore. In particular, since ROSAT stellar observations were often performed as snapshots with a certain time separation, we simulate analogous series of observations from the lightcurve of the Sun. With this aim in mind we have generated a set of observing times $\left(t_{0, i}\right)$ from the solar lightcurve. We take the solar intensity measured at $t_{0, i}$ and that measured at a later time corresponding to $t_{0, i}$ plus the explored time scale. For practical implementation we take the second observations after an interval corresponding to the time scale we are exploring with a tolerance of one day. If for example we are exploring the two month time scale, the first point is randomly taken from the solar lightcurve, while the second point is the solar intensity measured two months later with a tolerance of one day. For each pair of values generated in this way, we compute the observed variations as the ratio between the highest and lowest value. In order to avoid border effects, we have folded the lightcurve according to the 11 year solarcycle, and we have explored scales from one day to 11 years, spaced one day each and we have produced 10000 simulations for each explored time scale. For each scale we have derived the distributions of the amplitude variations, and measured the median and some percentile values, which for the AlMg filter observations are reported in Fig. 3.

The plot indicates that amplitude variations are strongly dependent on time scale, but that at each time scale a large spread is present. The highest probability to observe high amplitude variations is over a large time interval, between 4 and 7.5 years. At these time scales typically we observe variations of the order of 1 dex, but over the same time scales we observe also the largest spread, with $80 \%$ probability detecting variations in the $0.1-1.8$ dex range.

Some of the distributions of the amplitude variations computed above for some different time scales are shown in Fig. 4. The results for the Al filter are very similar. We have a probability of $50 \%$ to detect variations above a factor of two when we observe the Sun on time scale of the order or greater than one year, and variations by a factor of 10 when we observe the Sun on time scales in the 4-7.5 year range. 


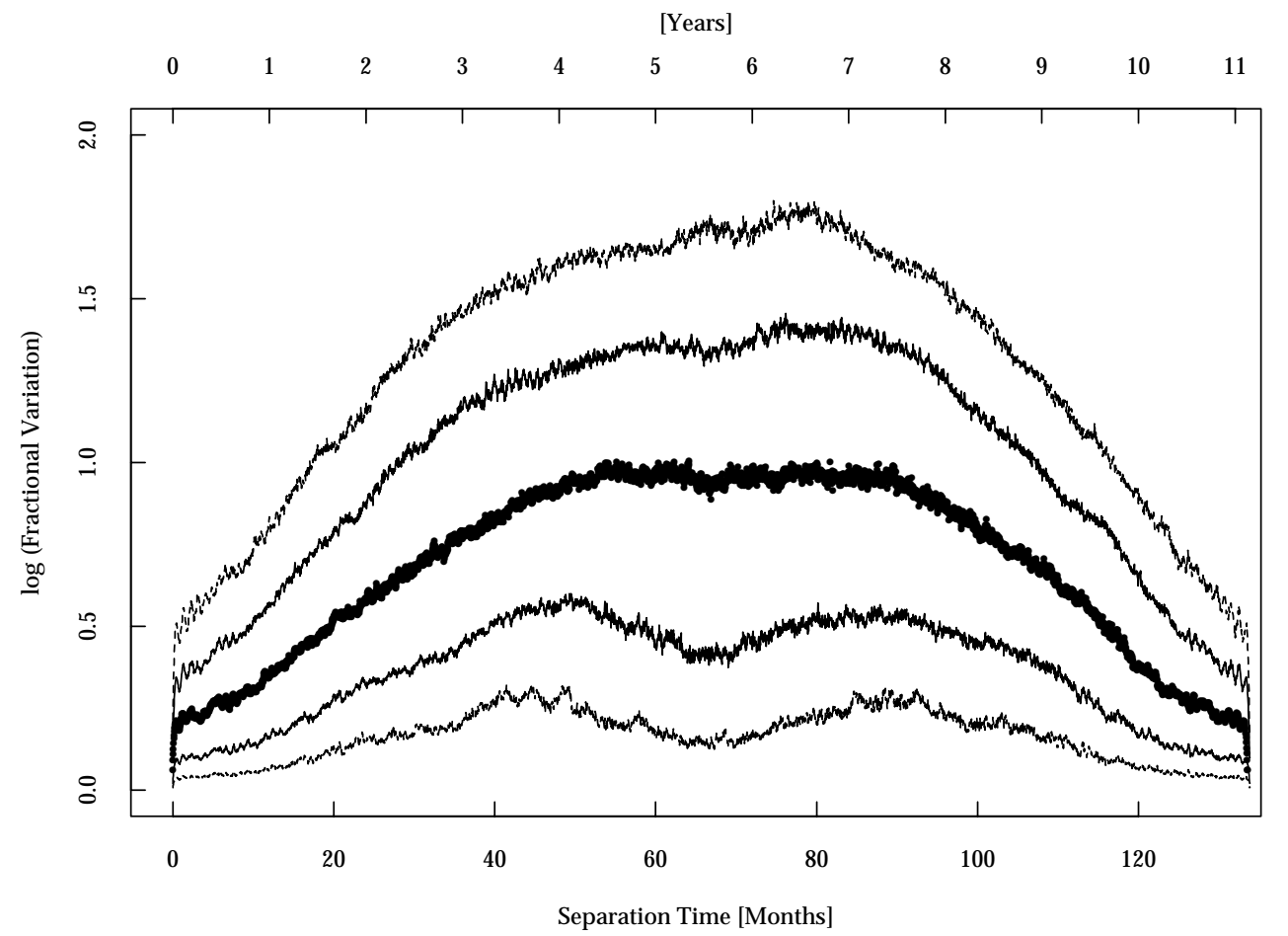

Fig. 3. Percentiles of the distributions obtained simulating pairs of solar AlMg observations as function of separation time. The marked solid line indicates the median, while the other lines correspond to the 10, 25, 75, and $90 \%$ percentiles.

\section{Comparison with nearby stars}

We have already compared (Sect. 2) the observed spread in the $\mathrm{X}$-ray luminosity of the Sun with that observed in the X-ray luminosity distributions of the nearby F- and G-type stars, concluding that solar-like variability can contribute at most to $60 \%$ of the observed spread. In this section we compare the variability properties of stars with those of the Sun. As mentioned earlier this comparison is not straightforward since the observational procedures differ. In particular ROSAT/PSPC operations were subject to a set of observational constraints that limited its capability to perform long continuous observations. As a consequence most of the exposures were fragmented in a number of temporal slots of the order of a few ksec and with a time separation of the order of the satellite orbit or more. Indeed the ROSAT/PSPC, because of its orbit, performed observations typically of a few ksec duration. The stellar observations that we consider here are hence a set of exposures of a few ksec each obtained either on contiguous spacecraft orbits or on orbits far away.

The energy bandpass is a further area of uncertainty, since solar variability properties depend on energy, and the bandpasses of the solar detectors are different from the stellar ones. In particular the Yohkoh bandpass (both with $\mathrm{Al}$ and AlMg filters) coincides approximately with the hard band of the ROSAT/PSPC (0.7-2.4 keV).

In order to compare the X-ray stellar variability with the solar one we use the sample studied by Marino et al. (2002) that includes F7-K2 main sequence stars in the solar neighborhood that have been detected in pointed ROSAT/PSPC observations. In their paper the authors analyze the variability properties of the sample on various time scales, and for the purpose of present paper we take the subsample of stars with at least two temporal segments with at least 30 counts each. Marino et al. (2002) evaluate the stellar variability properties in the $0.1-2.4 \mathrm{keV}$ band, using a conversion factor from counts to flux based on a single temperature plasma model, and negligible interstellar absorption. The assumed coronal temperature was estimated for each individual observation from the measured hardness ratio. Since the amplitude of X-ray variability depends steeply on the energy passband, in the present paper we have recomputed the conversion factors from stellar counts to flux, derived from each observed hardness ratio, in order to convert counts from the $0.1-2.4 \mathrm{keV}$ (the native PSPC bandpass) band to flux in the $0.7-2.4 \mathrm{keV}$ that better matches the Yohkoh instrumental response. The use of individual hardness ratios to evaluate stellar X-ray fluxes allow us to take into account both intensity and spectral variations. We have not attempted to extrapolate the solar flux to the PSPC bandpass, because this instrument has a large effective area in the soft band where Yohkoh/SXT has very low sensitivity. Since most of the stars have the bulk of their emission in the soft band, the extrapolation of solar flux observed with Yohkoh to PSPC band depends very critically on the accurate estimate of the emission measure distribution of solar plasma outside of the sensitivity range of the instrument, bringing a large uncertainty in the estimate solar flux in the PSPC energy bandpass.

For each star matching our criteria we have computed the $\mathrm{X}$-ray luminosity in the $0.7-2.4 \mathrm{keV}$ bandpass for each temporal segment and the amplitude variation as the ratio between the "instantaneous" luminosity and the minimum value measured for that star. We have associated with this amplitude variation a time scale that is the absolute value of the difference between 

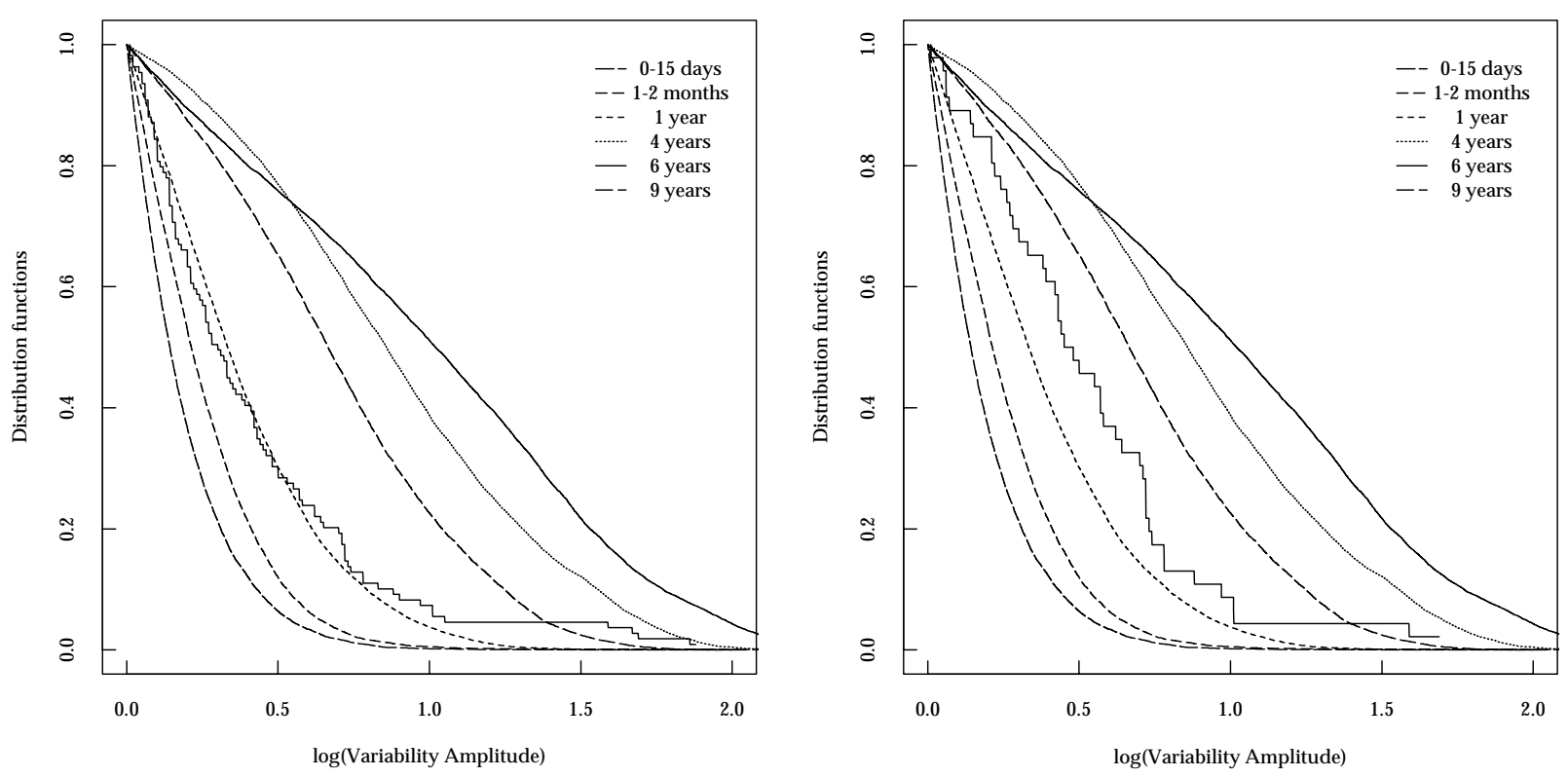

Fig. 4. Left panel: cumulative distribution of the amplitude variations obtained from the AlMg filter solar X-ray observations, when observed at different time scales. The legend in the figure indicates the explored time scales. The stepped curve is the cumulative distribution of the amplitude variations obtained for the nearby F7-K2 main sequence stars (109 data points) Right panel: same as in left panel, but with the cumulative distribution of the amplitude variations obtained for the nearby stars observed with time distance greater than 3 days alone (46 data points, stepped distribution).

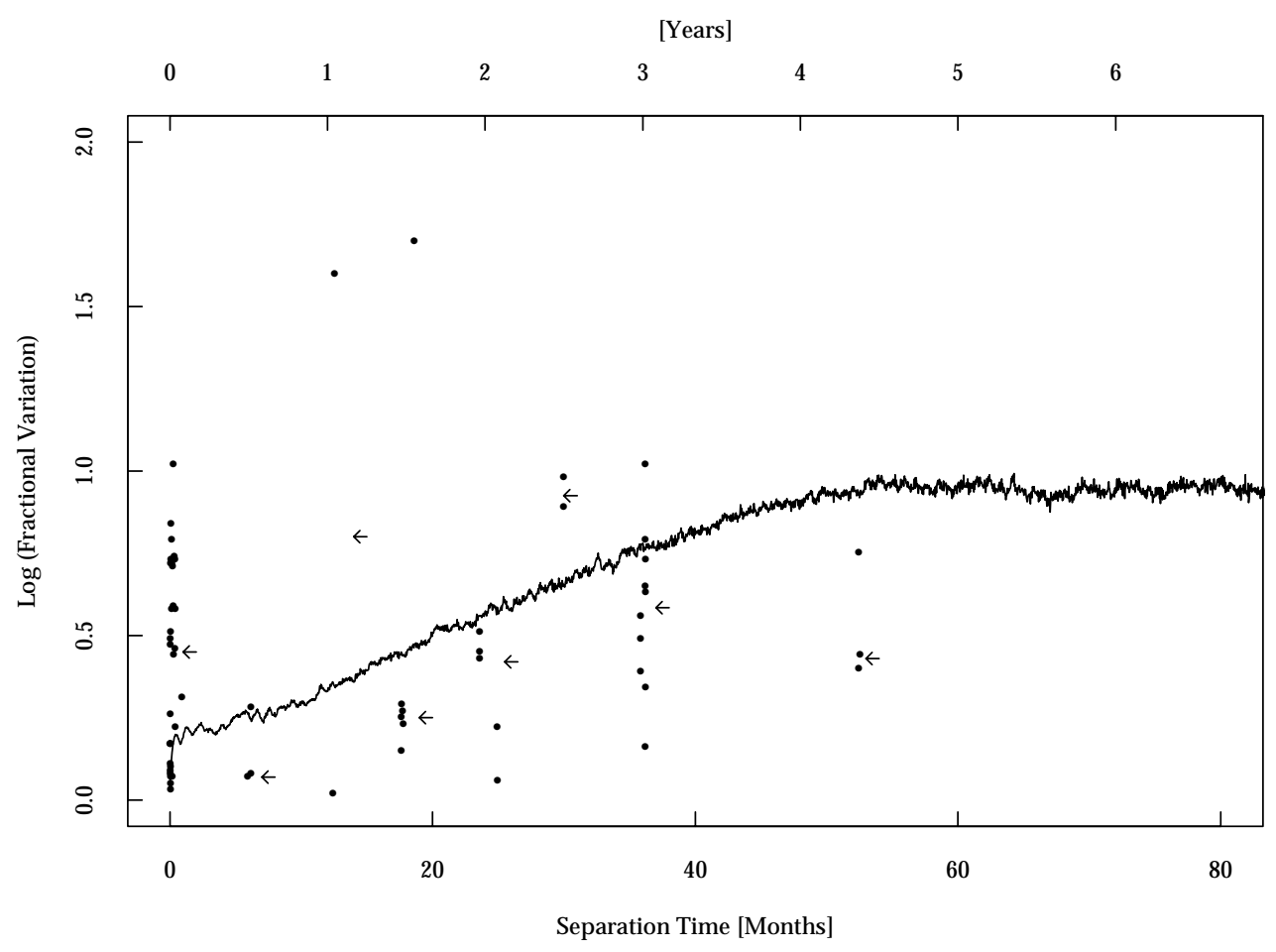

Fig. 5. The dots indicate the amplitude of X-ray variations observed in solar-type stars of Marino et al. (2002) in the band 0.7-2.4 keV vs. time scale. Arrows mark the median values of stars observed with time separation of the same order. The solid line marks the median value of the amplitude variations obtained from the AlMg Yohkoh observations of the Sun of Fig. 3.

each observation time and the time at which the minimum luminosity was observed.

In order to compare star properties with Sun's we compute the stellar cumulative distribution of the amplitude variations reported in Fig. 4 (stepped curve). The stellar distribution overlaps the curves obtained for the Sun at shortest time scales, consistently with the time scale distributions in stellar observations, dominated by short time scales $(\sim 50 \%<1$ day, $\sim 70 \%<$ 1 month). We repeated the same procedure for the stars observed with a time distance larger than 3 days (about $40 \%$ of the 


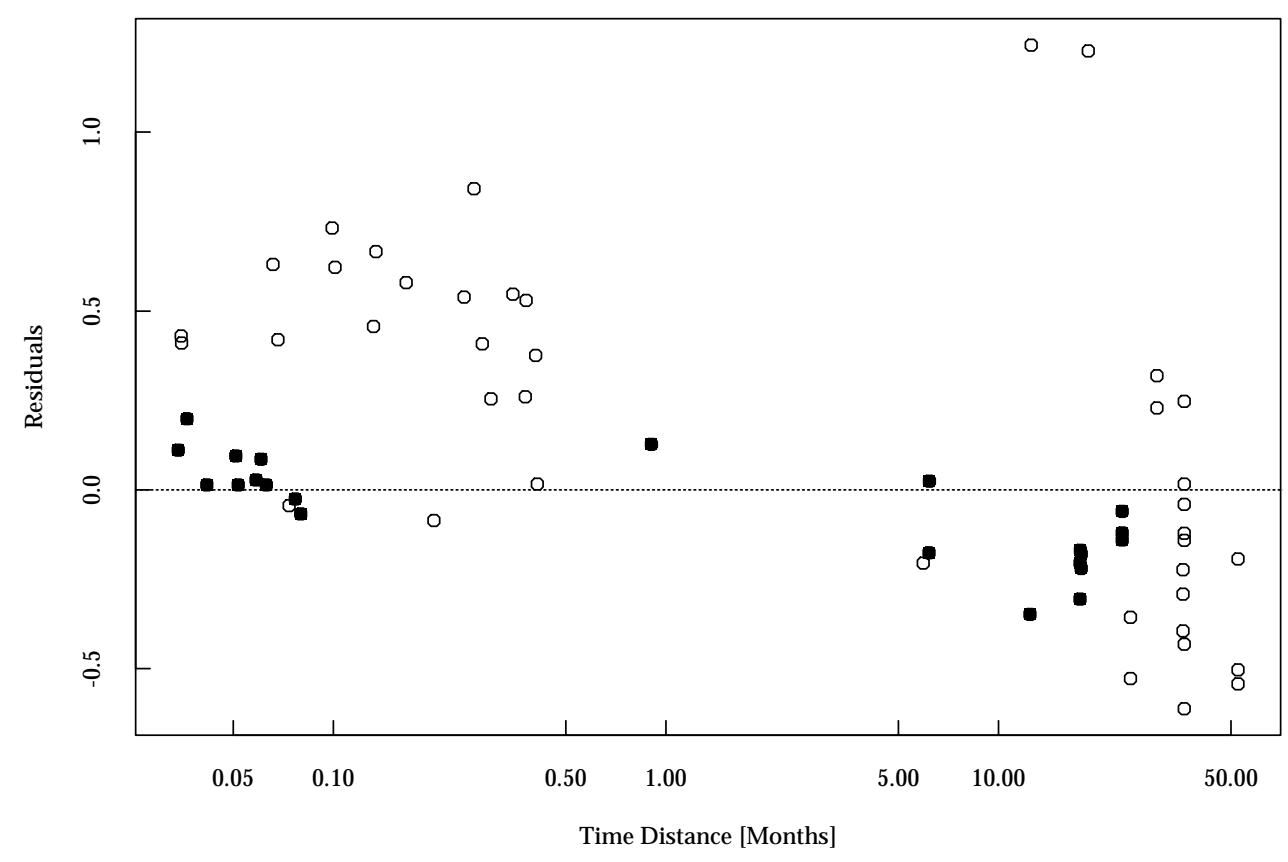

Fig. 6. Residuals of the stellar amplitude variations from the solar median of Fig. 3 for stars in our sample versus time scale. Empty points mark stars with $\bar{L}_{x}<10^{28} \mathrm{erg} / \mathrm{s}$ while filled points are stars with $\bar{L}_{x}>10^{28} \mathrm{erg} / \mathrm{s}$.

stellar observations) and found (Fig. 4, right panel) that the stellar distribution shifts towards larger amplitudes, qualitatively in agreement with the solar behaviour.

Assuming that the Sun is representative of the solar-type stars in the solar neighborhood we expect that half of the stars will be above the solid line representative of the median of the amplitude variations of the Sun in Fig. 3, and the remaining half below. The distribution of stars in this plane, explored with a time scale in the same range used for solar analysis, is shown in Fig. 5. About half ( 32 out of 62 points) are indeed below the median and the rest above, but the figure shows that at short time scale, stars tend to stay above the solar median (they are more variable than the median Sun), while at longer time scale they tend to be below the solar median (they are less variable than the median Sun). The enhanced variability observed on short time scales among the stars can be explained by the observational bias in the solar Yohkoh measurements, in which, as discussed in Sect. 1, the observational setup changed when a large flare occurred, producing a bias against the large flares. The effect is to underestimate the amplitude variations of the Sun at short time scales.

Since we know from Ca II measurements (Baliunas et al. 1995) that the properties of the activity cycle depend on the activity level, we report in Fig. 6 the residuals of the stellar amplitude variations from the solar median value versus time scale, separately for stars with $\bar{L}_{x}<10^{28} \mathrm{erg} / \mathrm{s}$ (empty points) and with $\bar{L}_{x}>10^{28} \mathrm{erg} / \mathrm{s}$ (filled points), where $\bar{L}_{x}$ has been derived dividing the total number of counts for the total exposure time obtained summing up all the available observations. The time in the plot is in a logarithm scale in order to better show the behavior at short time scales. We note that active stars show very small amplitude variations (within a factor of two) with all the points at time scales larger than one month below the solar median, i.e. they tend to be less variable than the Sun when we consider the same time scales.

Moderately active stars $\left(\bar{L}_{x}<10^{28} \mathrm{erg} / \mathrm{s}\right)$ show larger amplitude variations. Residuals of 6 out of the 20 stars observed with time separation larger than one month are positive, while the remaining 14 are negative, i.e. they are below the median value delineated by the Sun. These numbers seem to indicate that at least a fraction of less active stars can have cycles similar to that observed in the Sun. Of course our results are based on the assumption that stellar cycles have amplitudes and time scales similar to the solar one, and we cannot exclude that periods and amplitudes very different from the solar one can be present in all stars.

\section{Summary}

In this work we have analyzed Yohkoh solar X-ray synoptic observations in order to compare solar variability properties with stellar ones. Our analysis shows that:

- X-ray solar variations by a factor of two are common on short-medium time scales, while variations by a factor of three to four are due to long-term cyclic-like variability.

- At most $60 \%$ of the spread observed in nearby star X-ray luminosity distributions can be explained by solar-like variability.

- We have quantitatively determined how the solar X-ray variability changes with the time scale in the Yohkoh energy bandpass.

- The comparison with variability properties of nearby stars indicates that a fraction of moderately active stars $\left(\bar{L}_{x}<\right.$ $10^{28} \mathrm{erg} / \mathrm{s}$ ) can have properties similar to the Sun, consistent with the presence of X-ray cycles similar to the solar 
one, while the most active stars seem to lack these kinds of cycles.

Acknowledgements. We acknowledge financial support from ASI and MIUR. We thank F. Favata, S. Orlando, G. Peres, F. Reale and S. Sciortino for useful discussions and comments.

\section{References}

Baliunas, S. L., Donahue, R. A., Soon, W. H., et al. 1995, ApJ, 438, 269

Drake, J. J., Peres, G., Orlando, S., Laming, J. M., \& Maggio, A. 2000, ApJ, 545, 1074
Hempelmann, A., Schmitt, J. H. M. M., \& Stepien, K. 1996, A\&AS, 305,284

Kreplin, R. W. 1970, Ann. Geophys. 26, 367

Marino, A., Micela, G., Peres, G., \& Sciortino, S. 2002, A\&A, 383, 210

Morrison, M. 1994, Yohkoh Analysis Guide, Vol. 2: Reference Guide http://www.Imsal.com/SXT/html2/yag/uguide/ uguide.html

Pallavicini, R., Tagliaferri, G., \& Stella, L. 1990, A\&A, 228, 403

Peres, G., Orlando, S., Reale, F., Rosner, R., \& Hudson, H. 2000, ApJ, 528,537

Schmitt, J. H. M. M. 1997, A\&A, 318, 215

Stern, R. A. 1998, in Cool Stars, Stellar Systems, and the Sun, ed. R. A. Donahue, \& J. A. Bookbinder, ASP Conf. Ser., 154, 223 